\title{
Overview of current and emerging issues in endocrinological complications of thalassaemia
}

\author{
V. de Sanctis \\ Pediatric and Adolescent Outpatient Clinic, Quisisana Hospital, Ferrara, Italy
}

\begin{abstract}
Clinical advances in the treatment of thalassaemia major (TM) patients have helped to increase substantially the life expectancy of patients. The TM patients today represent the first generation of adult thalassemics. As patients enter puberty, they begin to experience a variety of endocrine abnormalities, presumably the results of chronic anaemia and tissue iron deposition from the chronic transfusion therapy. In patients with TM, the anterior pituitary gland is particularly sensitive to free radical stresses. Recent reports have documented a frequency of severe growth hormone deficiency in $13 \%-32 \%$ of adult patients with TM. The prevalence of impaired adrenal function in TM patients has been reported from 0 to $33 \%$, depends on the age of the population studied, although clinical adrenal insufficiency (AI) is rare.

Thyroid dysfunction has been observed in $13-60 \%$ of patients, but its severity is variable in different series. Acquired central hypothyroidism $(\mathrm{CH})$ is a rare complication.
\end{abstract}

\section{Introduction}

Clinical advances in the treatment of TM patients have helped to increase substantially the life expectancy of patients. The TM patients today represent the first generation of adult thalassemics. As patients enter puberty, they begin to experience a variety of endocrine abnormalities, presumably the results of chronic anaemia and tissue iron deposition from the chronic transfusion therapy.

Correspondence: V. de Sanctis, Pediatric and Adolescent Outpatient Clinic, Quisisana Hospital, Ferrara, Italy.

E-maio: vdesanctis@libero.it

Key words: endocrine complication, thalassemia.

(C) Copyright V. de Sanctis, 2011

Licensee PAGEPress, Italy

Thalassemia Reports 2011; 1(s2):e20

doi:10.4081/thal.2011.s2.e20

This article is distributed under the terms of the Creative Commons Attribution Noncommercial License (by-nc 3.0) which permits any noncommercial use, distribution, and reproduction in any medium, provided the original author(s) and source are credited.

Parts of this work were presented at the "12th International Conference on Thalassemia and Hemoglobinopathies", Antalya (Turkey), 11-14 May 2011.

\section{Adult growth hormone deficiency}

In patients with $\beta$-thalassemia major (TM), the anterior pituitary gland is particularly sensitive to free radical stresses. It has been reported that the GH deficiency (GHD) may be secondary to either pituitary or hypothalamic dysfunction. The duration of the disease, the patient's age and the severity of iron overload are the most important factors responsible for the defect of growth hormone (GH) secretion.

Recent reports have documented a frequency of severe growth hormone deficiency in $13 \%-32 \%$ of patients with $\beta$-thalassemia major. All of these patients underwent GH-releasing hormone (GH-RH) plus arginine (ARG) testing.

We found and a high prevalence of severe GHD in adult TM patients after glucagons stimulation test (GST). This percentage is higher compared to previous reports and may be related to the older age $(37.8 \pm 8.5$ years) of our patients and to the $\mathrm{GH}$ stimulation test employed in our study.

\section{Adrenal isufficiency}

There are only a few studies on adrenal function. A prevalence of impaired adrenal function in TM patients (from 0 to 33\%) depending on the age of the population studied, duration of blood transfusion, iron overload and procedures used for the evaluation of the diagnosis, has been reported in the literature, although clinical adrenal insufficiency (AI) is rare.

In our study, all TM patients had morning cortisol concentrations above $100 \mathrm{nmol} /$, considered a diagnostic value for ruling out hypothalamo-pituitary-adrenal insufficiency . But 17 out of 25 patients (68\%) had an impaired peak cortisol response after GST and one TM patient had peak cortisol response compatible with AI.

Thyroid Dysfunction

Thyroid dysfunction has been observed in $13-60 \%$ of patients, but its severity is variable in different series. Acquired central hypothyroidism $(\mathrm{CH})$ is a rare complication.

Here we report a TM patient with short stature and hypogonadism. $\mathrm{CH}$ associated with multiple medical complications was found. This case stresses the importance of routine thyroid function testing and interpretation of results, especially in the presence of clinical manifestations.

Acquired $\mathrm{CH}$ is a rare form of hypothyroidism secondary to various diseases.

The prevalence of $\mathrm{CH}$ in the general population is estimated at around 1/30,000-1/50,000 individuals. It may be isolated, or more commonly, associated with other pituitary hormone defects.

Acquired $\mathrm{CH}$ has been observed in 4 out of 1590 (0.25\%) TM patients, from May 1972 to October 2010, followed at the Day Hospital of Paediatric Endocrinology of St. Anna Hospital of Ferrara (De Sanctis $\mathrm{V}$, personal observation). However, the involvement of thyrotropic cells is probably more common. The diagnosis cannot be made with only TSH determination since in these patients TSH may be in the normal range. Biochemically there is low or normal TSH and low FT4 concentrations. These parameters must be simultaneously evaluated to avoid delay or error in diagnosis. 
Our recommendation is that if the level of FT4 is in the lower onethird of the normal range, then the patient should be started on levothyroxine (L-T4), but only after having excluded a concomitant adrenal insufficiency. If the level of free thyroxine is not consistently low, then we follow the patient frequently. In the management of $\mathrm{CH}$ patients the best way to monitor hypothalamic-pituitary-thyroid function is the measurement of serum FT4.

Pituitary hormone replacement therapy may require an adjustment of L-T4 treatment, as female patients under estrogen treatment and male patients with $\mathrm{GH}$ treatment will need a higher L-T4 dose in order to remain in the euthyroid range.

\section{References}

1. De Sanctis V, Skordis N, Galati MC, et al. Growth hormone and adrenal response to intramuscular glucagons test and its relationship to IGF-1 production and left ventricular ejection fraction in adult B thalassaemia major patients. Ped Endocrinol Review 2011, in press.

2. De Sanctis V, Giovannini M. Acquired central hypothyroidism in a male thalassaemic patient with severe iron overload. Ped Endocrinol Review 2011, in press. 